\title{
Punctuated equilibrium in an evolving bacterial population
}

\author{
Indranath Chaudhuri and Indrani Bose \\ Department of Physics, \\ Bose Institute, \\ 93/1, Acharya Prafulla Chandra Road, \\ Calcutta-700 009, India.
}

\begin{abstract}
Recently, Lenski et al have carried out an experiment on bacterial evolution. Their findings support the theory of punctuated equilibrium in biological evolution. We show that the $\mathrm{M}=2$ Bak-Sneppen model can explain some of the experimental results in a qualitative manner.
\end{abstract}

There are two major schools of thought in evolutionary biology: gradualism which implies continuous evolutionary changes and the theory of punctuated equilibrium (PE) which states that evolutionary activity occurs in bursts. Long periods of 'stasis' are followed by short periods of rapid changes. Recent exhaustive studies of fossil beds lend support to the second theory [1]]. In a remarkable but controversial experiment, Lenski et al [2, 3] studied an evolving bacterial population for approximately 10,000 generations. They inoculated a flask of low sugar broth with a dollop of bacteria. At the end of a day, a bit of the bacterial broth was siphoned into a fresh flask of food to keep the cells growing and dividing. Every 15 days, a sample bacterial population was frozen for later analysis. After four years, data for 10,000 generations were available. Lenski et al found evidence of PE when they measured the average cell size every 100 generations. The relative fitness, a measure of the increase in the growth rate of the descendant population over that of the ancestral population, also increases in a step-like manner. Further, the average cell size and the mean fitness appear to be correlated.

Lenski et al have interpreted the experimental results as suggesting that natural selection of rare beneficial mutations may be responsible for the punctuated growths seen in their experiments. During the periods of stasis, the bacteria are in a sense waiting for the beneficial mutations to occur. The rate of evolution is limited by the availability of such mutations. The bacterial 
(E. coli) population was allowed to develop from a single cell of a strain that can not exchange DNA. Spontaneous mutations are thus the only source of genetic change in the population. The experiment also involved competition for food because of the low availability of sugar. This constitutes a selection pressure on the bacterial population. There is a possibility that natural selection favours large cells as they have more energy reserve. The extra biomass can lead to faster replication than in the case of smaller cells. On the other hand, large cell size may simply be correlated with other traits which are the targets of natural selection and which are responsible for faster growth of the bacterial population.

Bak and Sneppen (BS) [4] have earlier proposed a model of biological evolution which exhibits $\mathrm{PE}$ in evolutionary activity in the so-called selforganised critical (SOC) state. There is a modified version of the BS model known as the M-trait BS model [5, 6] in which several biological species are considered, each of which is characterised by $M$ traits, instead of just one trait (fitness) as in the original BS model. In this paper, we show that the $\mathrm{M}=2 \mathrm{BS}$ model can explain the major results of Lenski et al's experiment in a qualitative manner.

In the BS model, each site of a one-dimensional (1d) lattice represents a particular species. We make a slight modification to this model. We divide the bacterial population into $N$ categories. Each category contains bacteria of similar characteristics. The $N$ categories correspond to the $N$ sites in the lattice. Two traits, namely, cell size and fitness (related to the replication rate), are associated with the population at each site. The dynamics of evolution is the same as in the $\mathrm{M}=2 \mathrm{BS}$ model. One assigns a random number (between 0 and 1) to each of the traits at all the $N$ sites. At each time step, the site with the minimum random value for a trait is identified. 'Mutation' occurs to bring about a change in the trait. The minimum random number is replaced by a new random number. The random numbers associated with any one of the traits of the neighbouring sites are also replaced by new numbers. This is to take into account the linkage of neighbouring populations in food chain. The last two steps are repeated and averages are taken for both the traits locally (over 40 sites) and globally (over 2000 sites). Unlike in the original BS model, we calculate quantities from the very beginning and not after the SOC state is reached. The relative fitness (RF) is defined to be the ratio of current fitness and initial fitness at time $t=0$. In the actual experiment, fitness is related to the growth rate of the bacterial population via 
replication. In the following, we compare the results obtained by simulation with the experimental results of Lenski et al. Fig.1 shows RF versus time (generations) for both experiment and simulation. In simulation, the RF is calculated for the whole bacterial population by taking a global average over all sites. Fig.2 shows the same curves but, now in the experiment, readings are taken every 100 generations, in contrast to 500 generations in Fig. 1. The step-like curve of the experiment is obtained in the simulation by taking a local average over 40 sites in calculating the RF. The data points are obtained every 100 time steps. Figs. 3 and 4 show results similar to those in Figs. 1 and 2 but now for the cell size (volume). Figs. 1 and 3 show that the RF and the average cell size increase quite rapidly in the first 2000 generations in the experimental environment. After several thousand generations, changes are imperceptible. The data points in the two Figures are taken every 500 generations. Figs.2 and 4 exhibit data taken every 100 generations. The step-like features correspond to periods of stasis followed by short periods of rapid evolution. The time scale over which data is obtained seems to be the deciding factor in the observation of PE. Punctuation shows up only when the RF and the average cell size are measured every 100 generations. At the longer time scale of 500 generations, the growths appear to be smooth and gradual. Objection has been raised [8] that if one looks closely enough at anything, steps will eventually be seen. In the BS model, gradual changes are seen when global averages are taken and steps appear for local averages. Fig. 5 shows the correlation between average cell size and mean fitness for both experiment and simulation. The correlation coefficients [7] are $\mathrm{r}=$ 0.954 (experiment), $\mathrm{r}=0.998$ (simulation).

From Figs. 1-5, it appears that the experimental and simulation results are in qualitative agreement. Many issues, however, have to be clarified before a quantitative agreement can be obtained. As already mentioned, Lenski et al have interpreted their experimental results by assuming that natural selection favours beneficial mutations which confer competitive advantage. In the BS model, mutations can be both beneficial and disadvantageous. In the first case, the smallest fitness value is replaced by a larger value. In the second case, the new fitness value is lower than the first one. Simulation of the BS model, however, shows an overall increase of the RF (or the cell size) in time. This is because the probability of beneficial bacterial mutation is more in the BS model. In fact, in the SOC state, most fitness values are above a critical threshold value. Consider that in a particular time step, the minimal 
fitness value is 0.2 . This number is to be replaced by a random number drawn from a uniform distribution over the interval $0-1$. Thus the probability that the new fitness value is less than the original one is 0.2 and the probability that the fitness increases is 0.8. Over a large number of generations, the average fitness (or the cell size) has a tendency to increase. Comparison of experimental and simulation results, however, shows that the cell volume, obtained from simulation, has a much smaller rate of growth than in the actual experiment. Also, the number of steps in the cell size is larger than as seen in the experiment. The experimental data for the RF (Fig.2) show four significant steps: 0 to 200, 300 to 500, 600 to 1200 and 1300 to 2000 . The steps in the case of the cell size (Fig.4) occur in the regions 0 to 200, 300 to 500, 600, 700 to 1100,1200 and 1300 to 3000. Thus there are only two discrepancies among the step regions in the cases of the RF and the cell size. The discrepancy is more in the case of simulation.

The relationship between simulation time steps and bacterial generations is not clear. In the experiment, about $10^{6}$ mutations occurred every day and there were approximately 6.6 generations per day. In the lattice model, the bacterial population has a coarse-grained representation. Bacteria of similar characteristics are grouped in a single category. So one mutation in the lattice model is equivalent to a large number of mutations in reality. In the experiment, serial transfer of a part of the bacterial poulation to fresh sugar medium occured at the end of a day. This feature is not explicit in the lattice model. Increase of the fitness parameter indicates a higher replication rate and so the population increase is taken into account in an indirect manner. One crucial feature of the experiment is the scarcity of food (sugar). Again, this feature is not inherent in the lattice model. The increase of the average cell size, over time, in the lattice model can, however, be considered to be an outcome of low food supply. The BS model has to be suitably modified to take into account the effect of competition for food. Also, the implication of the local versus the global average, in an actual experiment has to be understood. The SOC state of the BS model is characterised by power-law correlations [目] in space and time. The growth of the RF and the cell size, however, occur before the SOC state is reached. The SOC state corresponds to the saturation regions in the growth curves. Bak and Sneppen have shown that this state exhibits PE in the biological activity and has the characteristic that all mutations involve changing of fitness values below a self-organised critical value. Also, the distribution of distances between successive mutations has 
a power-law decay as a function of the distance between the mutations. The size and lifetime distributions of avalanches of successive mutations have a power-law form. Further experiments on bacterial evolution can test the validity of some of these ideas.

\section{Acknowledgement}

We thank Richard E. Lenski for permission to reproduce the original figure in Refs. [2] and [3]. One of the author I.C. is supported by the Council of Scientific and Industrial Research, India under Sanction No. 9/15(173)/96EMR-I. 


\section{Figure Captions}

Fig.1 Relative fitness versus time in experiment [3] and simulation. A global average is taken over 2000 sites of the lattice to obtain the data points in simulation.

Fig.2 Relative fitness versus time in experiment [3] and simulation. A local average is taken over 40 sites in simulation. the experimental data points are taken every 100 generations.

Fig.3 Average cell size versus time in experiment [3] and simulation (global average).

Fig.4 Average cell size versus time in experiment [2] and simulation (local average).

Fig.5 Average fitness versus average cell size in experiment [2] and simulation. 


\section{References}

[1] R.A.Kerr, Science 267, 1421 (1995)

[2] S.F.Elena, V.S.Cooper and R.E.Lenski, Science 272, 1802(1996)

[3] R.E.Lenski and M.Travisano, Proc. Natl. Acad. Sci. 91, 6808 (1994)

[4] P.Bak and K.Sneppen, Phys.Rev.Lett. 71, 4083 (1993)

[5] P.Bak, 'How nature works : the science of self-organised criticality' (Springer-Verlag, New York, 1996)

[6] S.Boettcher and M.Paczuski, Phys.Rev.Lett. 76, 348 (1996)

[7] S.C.Pearce, 'Biological Statistics : An Introduction' (McGraw-Hill, New York (1965))

[8] C. Mlot, Science 272, 1741 (1996) 

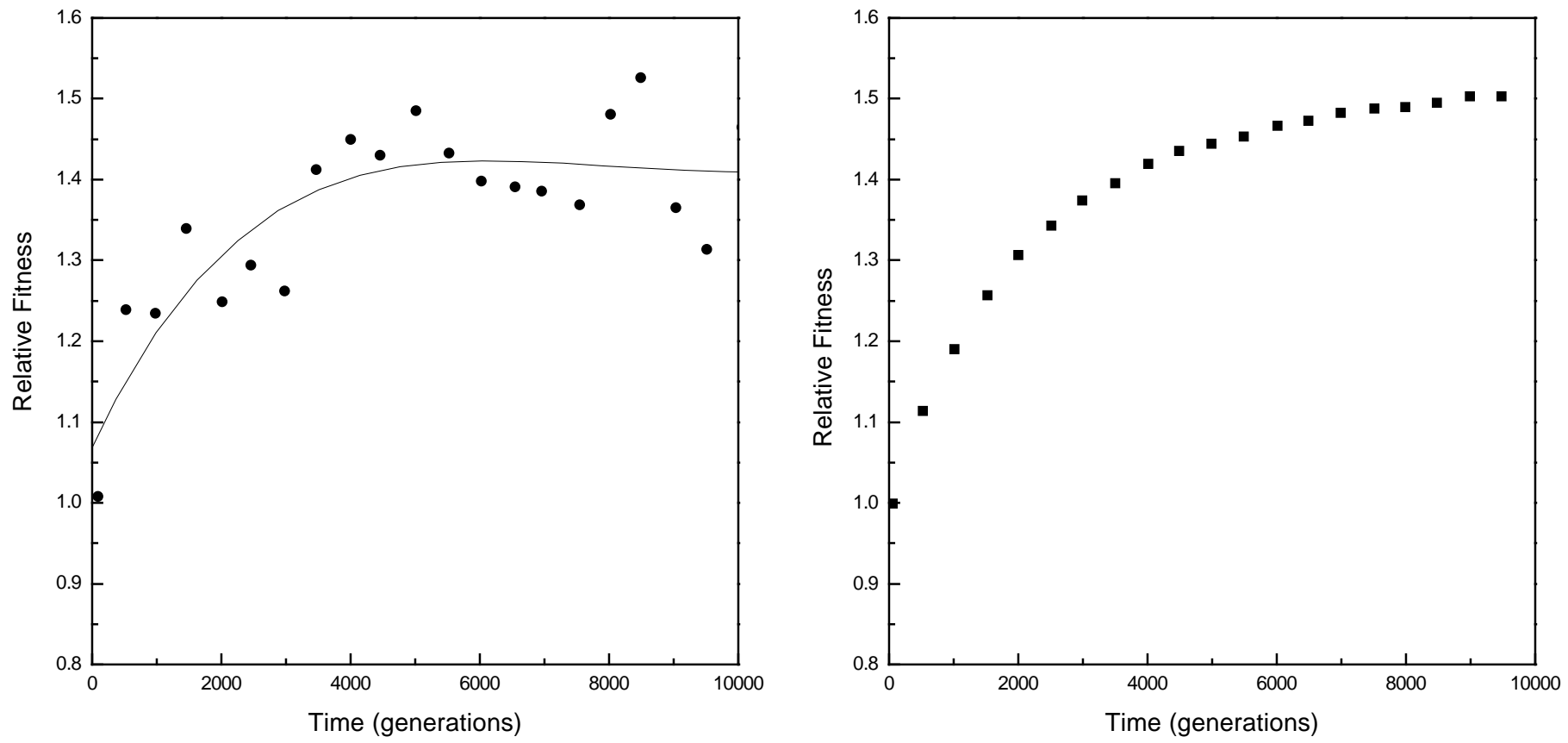

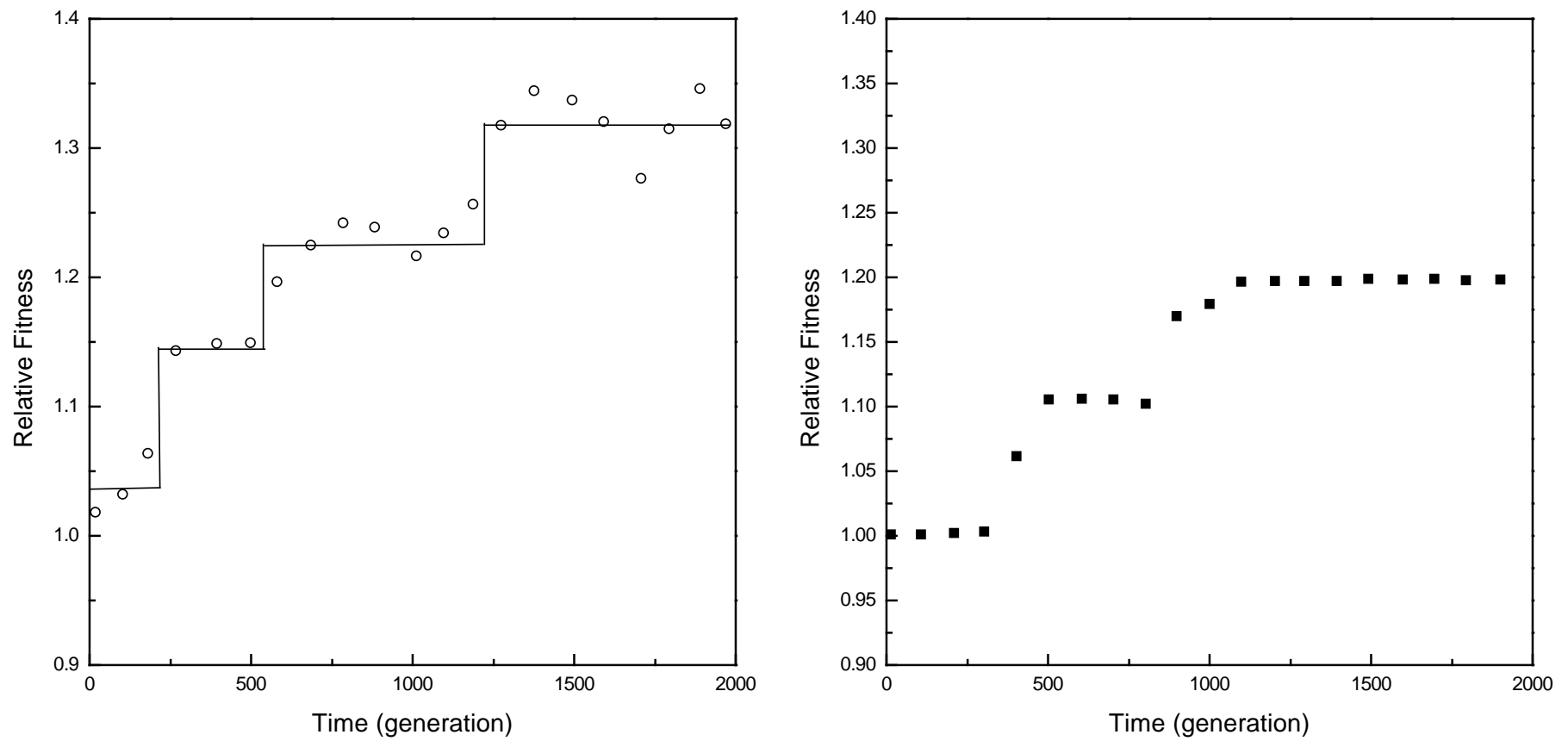

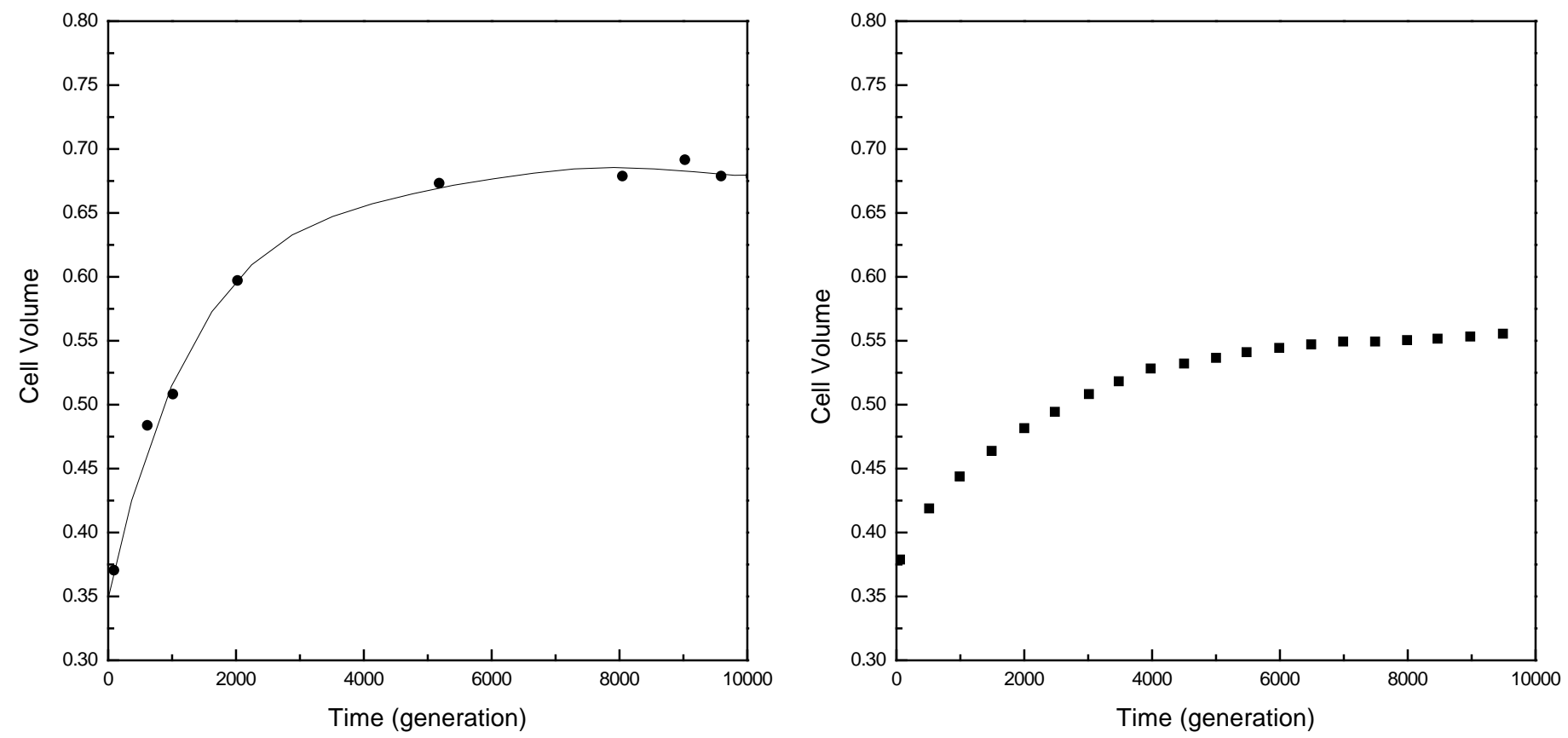

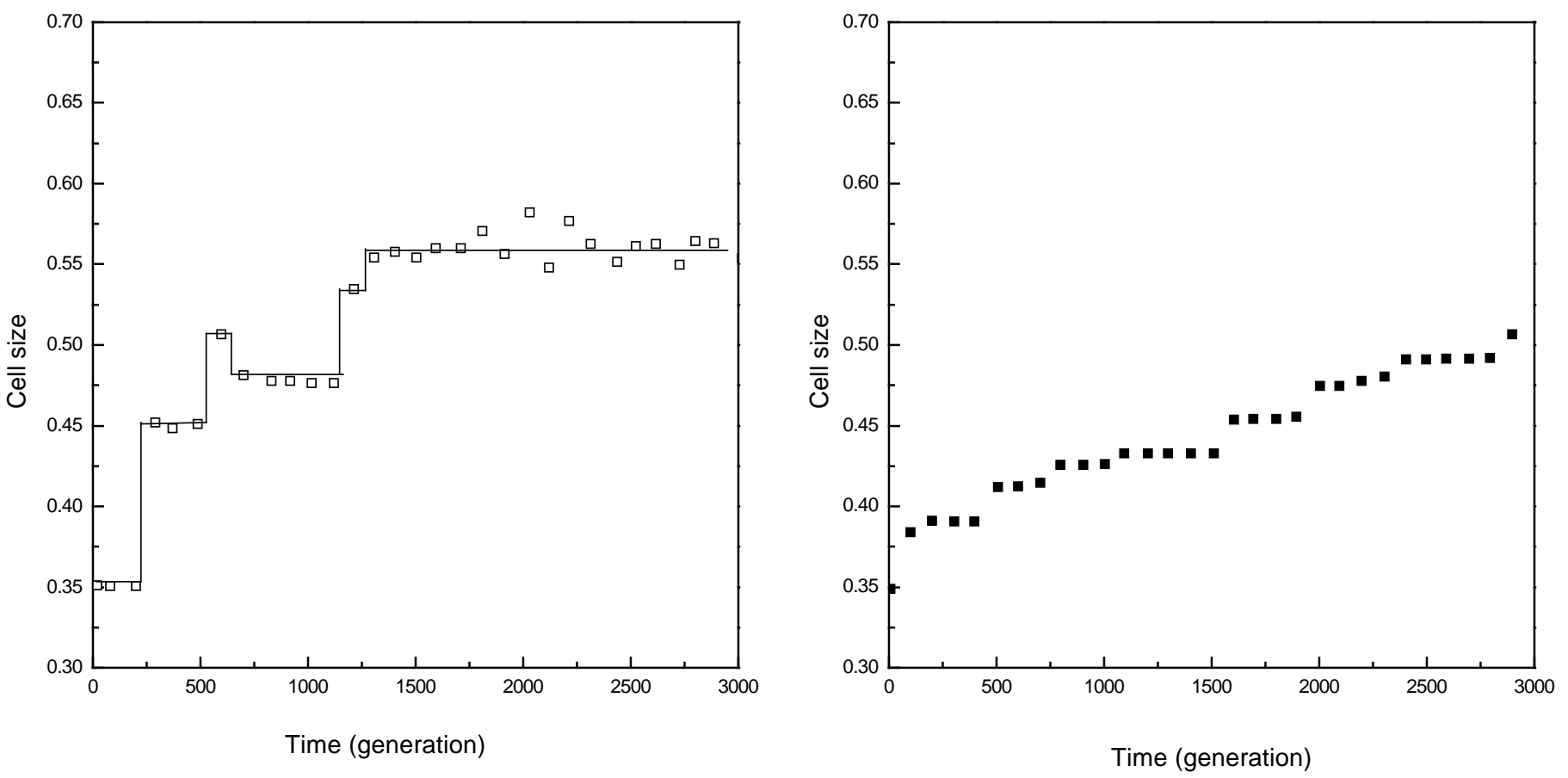

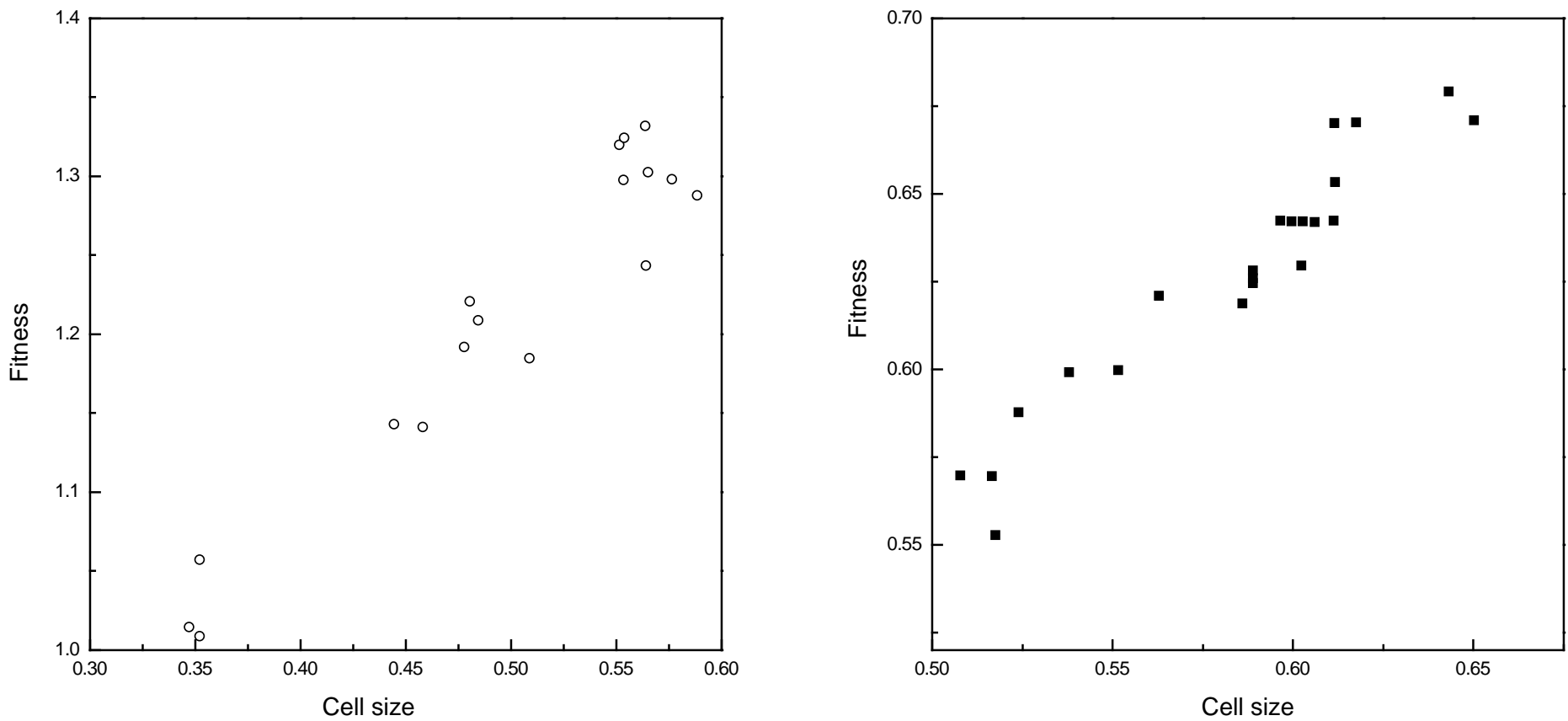